\title{
Tumor-like lesions of the heart valve
}

\author{
Venkatesh Krishnamurthy Tekur ${ }^{1}$, Sriarun Venkatesh Tekur ${ }^{2}$ \\ ${ }^{l}$ Consultant and Interventional Cardiologist, Apollo Hospitals, Opp IIM, Bannerghatta Road, Bangalore- \\ 560076 Karnataka, India. \\ ${ }^{2}$ Intern, JSS Hospital, Mahatma Gandhi Road, Mysuru, 570004 Karnataka, India.
}

\begin{abstract}
Valvular tumor and tumor-like lesions are rare. Fibroelastoma is the most common valvular tumor followed by myxoma. Atypical valvular lesions, such as organized thrombus, valvular calcification and valvular abscess may develop symptoms and present morphological features similar to those of the valvular tumors. The Author here reports a case of a tumour or tumour- like lesion over the mitral valve detected in a asymptomatic patient on preoperative evaluation prior to bilateral total knee replacement surgery for osteoarthritis.

Key-words: Valvular tumour, Tumour-like lesions,

Key Messages: Patients should be counselled about the high risk of stroke when tumour or tumour-like lesions are visualised on echocardiogram and encouraged to have a definitive diagnosis established by surgery and biopsy.
\end{abstract}

\section{Case History}

A 60-year lady asymptomatic cardiac wise was admitted for bilateral total knee replacement for osteoarthritis. On pre-operative cardiac evaluation, ECG showed non specific ST-T changes , and trans- thoracic echocardiogram showed a tumor mass between left atrium and left ventricle, with an echo-density somewhat greater than that of the myocardium.[fig 1].

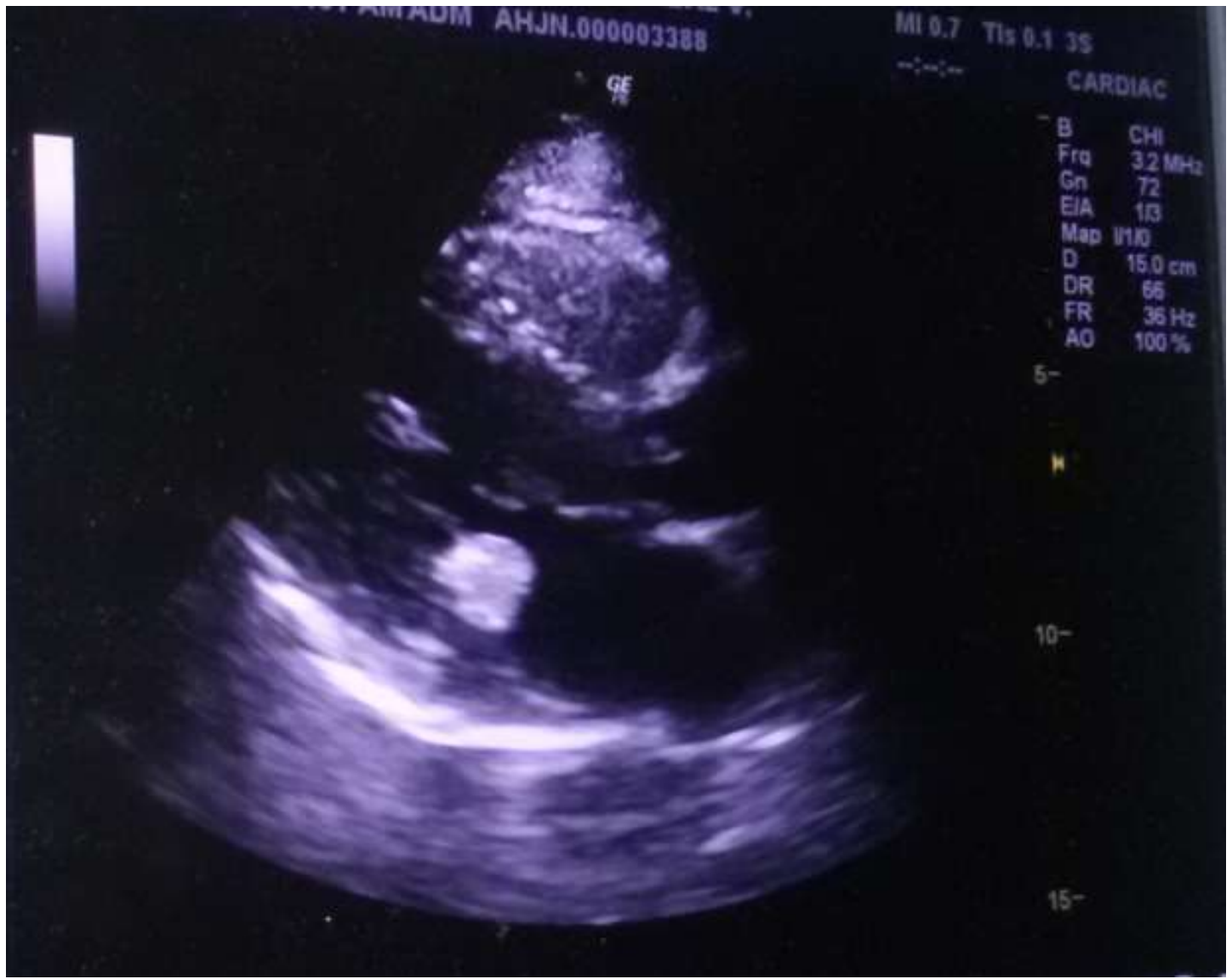

FIG.1. Transthoracic echocardiogram showing tumour mass between left atrium and left ventricle, with an echo-density greater than that of the myocardium. 
The mass measured around $1.43 \times 1.68 \mathrm{~cm}$ and appeared to be situated at the base of the posterior mitral valve leaflet.[fig2].

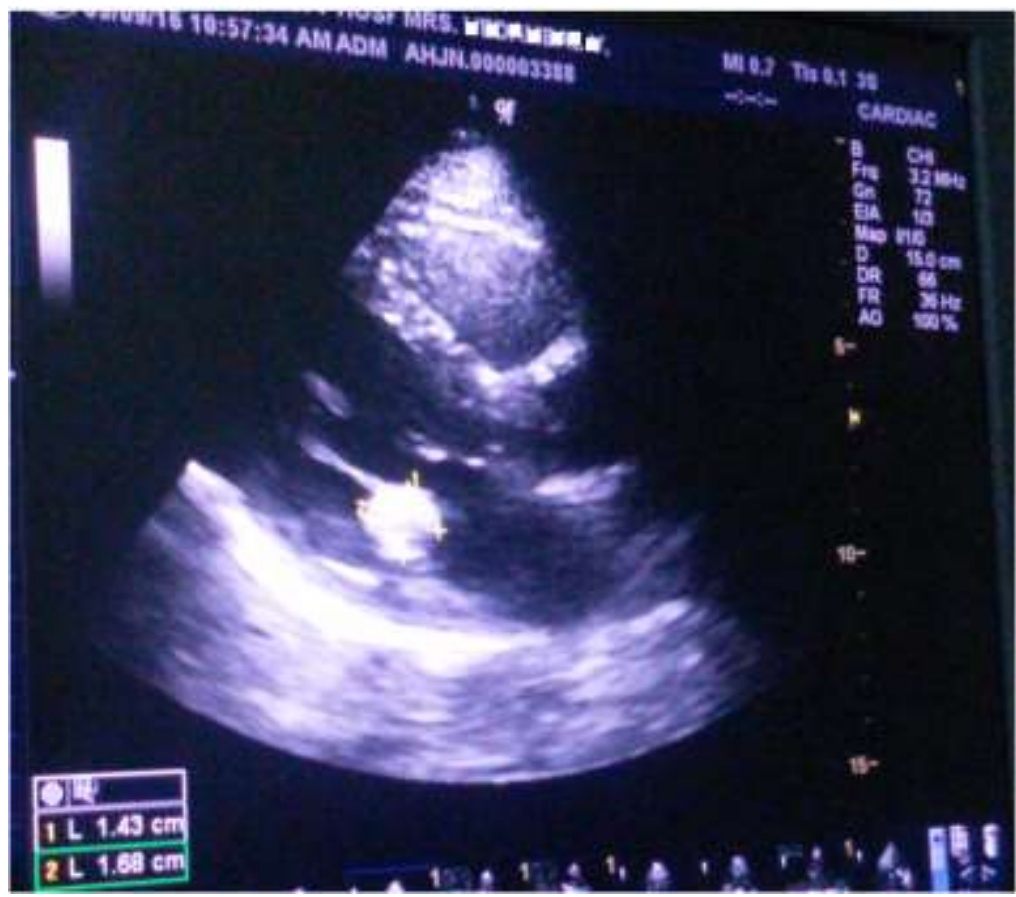

FIG.2. Trans thoracic echocardiogram showing almost circular mass situated over the base of posterior mitral leaflet.

A trans-oesophageal echocardiogram was performed. It confirmed trans- thoracic echocardiogram findings and the movement of the tumor mass was concordant with the movement of mitral valve ring. It was situated on the ventricular side of the posterior mitral valve. A thickening of the posterior mitral valve leaflet was noted. The mass was not infiltrating atrial or ventricular myocardium.[FIG 3].

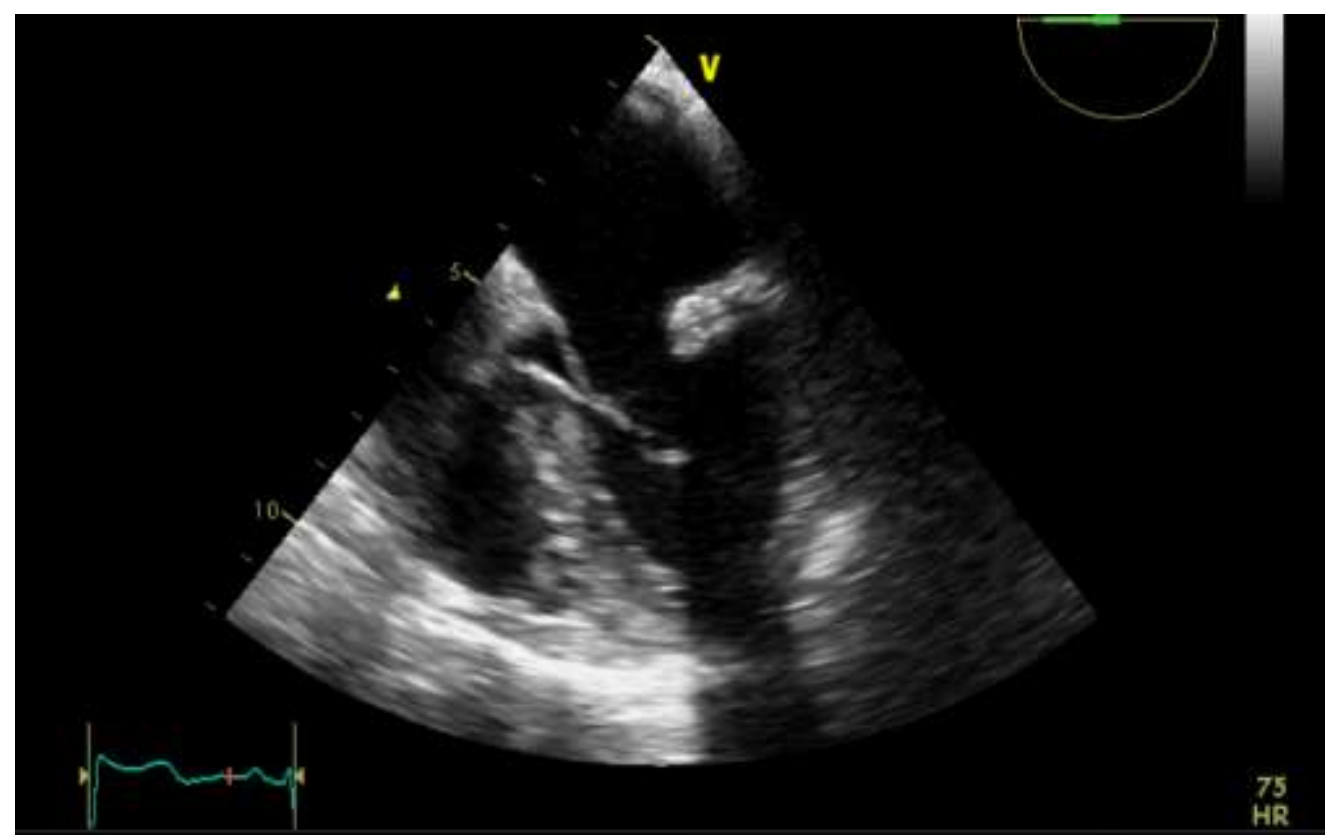

FIG.3. Trans oesophageal echocardiogram of the tumour mass.

There was mild mitral regurgitation. Patient refused to undergo any further cardiac investigations and preferred to go ahead with bilateral total knee replacement accepting moderate risk for cardiovascular events including stroke due to thromboembolism. 


\section{Discussion}

Yater was the first to describe valvular tumors in $1931 .{ }^{1}$ Fibroelastoma is the most common valvular tumor, followed by myxoma. Organized thrombus, valvular calcification and valvular abscess may mimic morphological features of valvular tumors. Valvular tumours are characterized by a smaller size, greater mobility, and more significant tendency to embolize by way of tumor fragments or thrombus formed around the tumor than the intramural tumors. ${ }^{2}$. The majority of valvular tumors are papillary fibroelastomas, with aortic valve being the most commonly affected valve $(52 \%)$, followed by the mitral valve $(16 \%){ }^{3}$. They predominantly affect cardiac valves and account for most cases of valvular tumor. Valvular myxomas are exceedingly rare accounting for $8.8 \%$ of cardiac myxomas. ${ }^{4}$. Valvular tumors and tumor-like lesions place the patients at a high risk of stroke in different ways. It is important to have an exhaustive differential diagnosis when confronted with tumour or tumour-like lesions over the cardiac valves. Calcification of the mitral valve annulus(mitral annular calcification-MAC), thrombus, and infective vegetations need to be considered. Caseous calcification of the mitral annulus (CCMA) is a rare variant of mitral annulus calcification (MAC), constituting about $0.63 \%$ of all mitral annular calcification cases $^{5}$. In cases of CCMA, echocardiography usually reveals a large, round, echodense mass with smooth borders and central areas of echolucency. ${ }^{6}$. Mitral valve abscess is another important differential diagnosis. In extreme cases of mitral valve abscesses, definite diagnoses can only be obtained during surgery. ${ }^{7}$ In summary, surgery with biopsy of the lesion would yield a definite diagnosis, as a tumour can be mistaken on echocardiogram for an atypical lesion like an abscess or a vegetation on biopsy .Patients should be counselled about the high risk of stroke when tumour or tumour-like lesions are visualised on echocardiogram and encouraged to have a definitive diagnosis established by surgery.

\section{References}

[1]. Historical reference: http://www.utmem.edu/cardiology/ppt/Sea\%20anemone.ppt\#279,10.

[2]. Edwards FH, Hale D, Cohen A, et al. Primary cardiac valve tumors. Ann Thorac Surg. 1991;52:1127-31.

[3]. Ngaage DL, Mullany CJ, Daly RC, et al. Surgical treatment of cardiac papillary fibroelastoma: a single center experience with eighty-eight patients. Ann Thorac Surg. 2005;80:1712-8

[4]. Wold LE, Lie JT. Cardiac myxomas: a clinicopathologic profile. Am J Pathol. 1980;101:219-40.

[5]. Harvinder Arora, Pankaj Madan, Leo Simpson, and Raymond F. Stainback. Caseous Calcification of the Mitral Annulus. Tex Heart Inst J. 2008; 35(2): 211-213.

[6]. Harpaz D, Auerbach I, Vered Z, Motro M, Tobar A, Rosenblatt S. Caseous calcification of the mitral annulus: a neglected, unrecognized diagnosis. J Am Soc Echocardiogr 2001;14 (8):825-31.

[7]. Sowa S, Kleinrok A, Gburek T, Zaremba-Flis E. Abscess of the mitral annulus mimicking left atrial tumour - case report. Kardiol Pol. 2007;65:571-4. 